\title{
Combined Effect of Pre-Strain at Elevated Temperature and Pre-Aging on Aging Strength and Microstructures of AA6082
}

\author{
Zhenhai Xu, Ru Chen and Debin Shan* \\ National Key Laboratory for Precision Hot Processing of Metals, Harbin Institute of Technology, Harbin 150001, P.R. China
}

With the increasingly serious energy and environmental issues, aluminum alloys are becoming increasingly desirable for transportation facilities due to their high specific strength. Their low formability results in the difficulty of forming complex-shaped panels and bodies. Hot stamping is a new technology developed to address this formability challenge by applying plastic deformation to the heated aluminum alloy sheet at the solution heat treatment temperature. The stamped panels gain high strength via the sequent aging treatment. Different panels, even different regions on the same panel, are subjected to different forming strains. The present paper is focused on the experimentally investigating the effects of the pre-strain at elevated temperature and pre-aging on the mechanical behavior and microstructures of AA6082 after the natural aging, paint-bake treatment, and artificial aging. Sample sheets of AA6082-T6 were pre-strained by uniaxial tension at different levels of strain, and then subjected to the pre-aging, natural aging, paint-bake treatment or artificial aging, respectively. The yield strength, tensile strength and elongation of post-aged samples were gained via the standard uniaxial tensile tests. The results show that both of the pre-strain at elevated temperature and pre-aging suppress the natural aging of AA6082. Pre-strain at elevated temperature induces weak bake softening, and has a detrimental effect on the artificial aging strengthening, while the sequent pre-aging could compensate the strength decrease.

[doi:10.2320/matertrans.MT-ML2019010]

(Received August 5, 2019; Accepted October 1, 2019; Published November 15, 2019)

Keywords: aluminum alloys, pre-strain at elevated temperature, pre-aging

\section{Introduction}

With the ever-increasing concern about the energy and environment crises, the aluminum alloy as a lightweight material is becoming increasingly desirable for various transportation facilities and movable equipment due to its high specific strength, as well as other attractive properties such as corrosion resistance, dimensional stability, etc. ${ }^{1)}$ However, aluminum alloy sheets have lower formability than conventional steel sheets, resulting in the difficulty of forming complex-shaped panels and bodies via the traditional cold stamping. In order to address this formability challenge in large batch production, various heat treatment methods have been integrated into the stamping process, resulting in the creation of heat-treatment-stamping integrated techniques, including intermediate annealing-stamping integrated method, ${ }^{2}$ ) warm stamping, ${ }^{3)}$ stamping in the $\mathrm{W}$ temper ${ }^{4}$ and hot stamping. ${ }^{5)}$ The latter one applies plastic deformation to the aluminum alloy sheet at the solution heat treatment temperature, and then quenches the formed part in cool dies. Therefore, the hot stamping is also called solution-heattreatment, forming and in-die quenching (HFQ) process. ${ }^{6}$ The following heat treatment plays an important role on the mechanical properties of the formed parts. Most research work is focused on the effects of the temperature, holding time and cooling rate. While the effects of other factors such as the heating rate and pre-strain on the heat treatment have begun to attract researchers' attention. ${ }^{7,8)}$ Pre-strain has been reported to have a substantial effect on the precipitation behavior of Al-Mg-Si alloys. ${ }^{9}{ }^{10)}$ The dislocations introduced by pre-strain are believed to act as sink of quenched-in vacancies and avoid clustering at room temperature, thus suppress the negative effect of natural aging. While during the paint-bake cycle, the dislocations could provide

*Corresponding author, E-mail: d.b.shan@gmail.com heterogeneous nucleation sites for the Guinier-Preston (GP) zones that easily grow and transform into $\beta^{\prime \prime}$ phase by the enhanced atomic transport. ${ }^{11,12)}$ It is also indicated that the pre-aging treatment imposes a significant effect on the precipitation hardening behavior of aluminum alloy. ${ }^{13,14)}$ Most work has been focused on the pre-strain at room temperature, while the combined effect of pre-strain at elevated temperature and pre-aging on the final aging strength and microstructures needs to be revealed.

In this paper the tensile samples were subjected to the pre-strain at the solution temperature, and then underwent the natural aging, paint-bake treatment or artificial aging, respectively, finally were subjected to the uniaxial tension to test the yield stress, tensile stress and elongation. The effect of pre-strain at elevated temperature and sequent aging on the mechanical behavior of AA6082 was analyzed.

\section{Experimental Procedure}

A commercial AA 6082 sheet with a thickness of $1.6 \mathrm{~mm}$ was used. The experimental procedure is as follows: The sheet samples were sheared into $10 \mathrm{~mm}$ wide tensile bars at $90^{\circ}$ to the rolling direction as shown in Fig. 1. To keep the uniform for different tensile bars with the same level of pre-strain, a pair of special high temperature grips as shown in Fig. 2 were developed. It has wide shoulders to contain several bars at the same time. The maximum number is up to 9 . The solution heat treatment was carried out at $540^{\circ} \mathrm{C}$ for 40 minutes in the furnace chamber of a universal mechanical testing machine. Uniaxial tensile pre-strain was conducted at an initial strain rate of $10^{-3} \mathrm{~s}^{-1}$. Pre-strains were introduced as levels of $5 \%, 8 \%$, and $13 \%$ in engineering strain measured. All samples were subjected to isothermal heat-treatments in an air furnace. Pre-aging was carried out at $140^{\circ} \mathrm{C}$ for 10 minutes just after pre-strain at elevated temperature. Paint-bake treatment and artificial aging were 


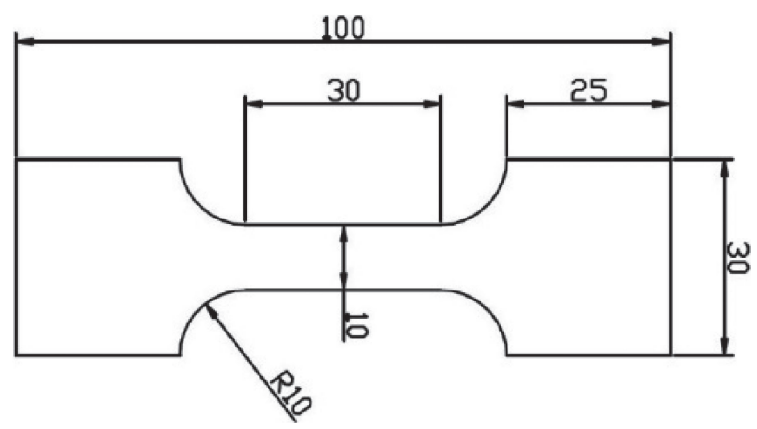

Fig. 1 Dimensions of the tensile bar.

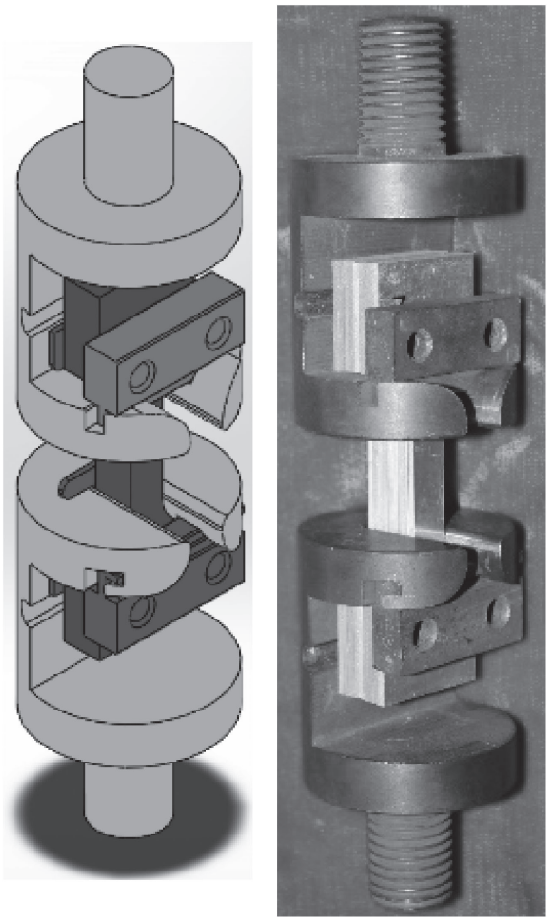

Fig. 2 A pair of special high temperature grips.

carried out at $180^{\circ} \mathrm{C}$ for 30 minutes and 6 hours, respectively. Post-aging mechanical properties testing was performed at room temperature in the rolling direction using the same strain rate as applied in the pre-straining process.

\section{Results and Discussions}

Figure 3 shows the effect of pre-strain at elevated temperature and pre-aging on mechanical properties of AA6082 after the natural aging of two weeks. The yield strength $\sigma_{\mathrm{s}}$, tensile strength $\sigma_{\mathrm{b}}$ and elongation $\delta$ of samples after the natural aging are $166 \mathrm{MPa}, 290 \mathrm{MPa}$ and $20.8 \%$, respectively. While after introducing the pre-strain at elevated temperature, the strength of the pre-strained samples was lower than that of un-deformed samples under each strain level. With increasing the pre-strain level, both $\sigma_{\mathrm{s}}$ and $\sigma_{\mathrm{b}}$ decrease, while $\delta$ increases. It is fair to claim that the prestrain treatment could reduce the natural aging. While after introducing the pre-aging following the pre-strain, both $\sigma_{\mathrm{s}}$ and $\sigma_{\mathrm{b}}$ decrease further at each pre-strain level than that of deformed samples without pre-aging. It indicates the

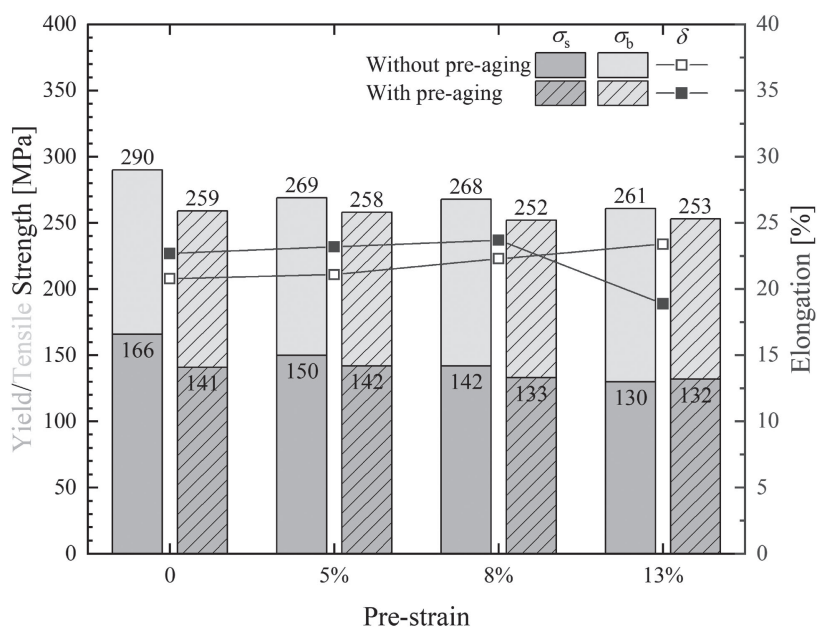

Fig. 3 Effect of pre-strain at elevated temperature and pre-aging on mechanical properties of AA6082 after natural aging.

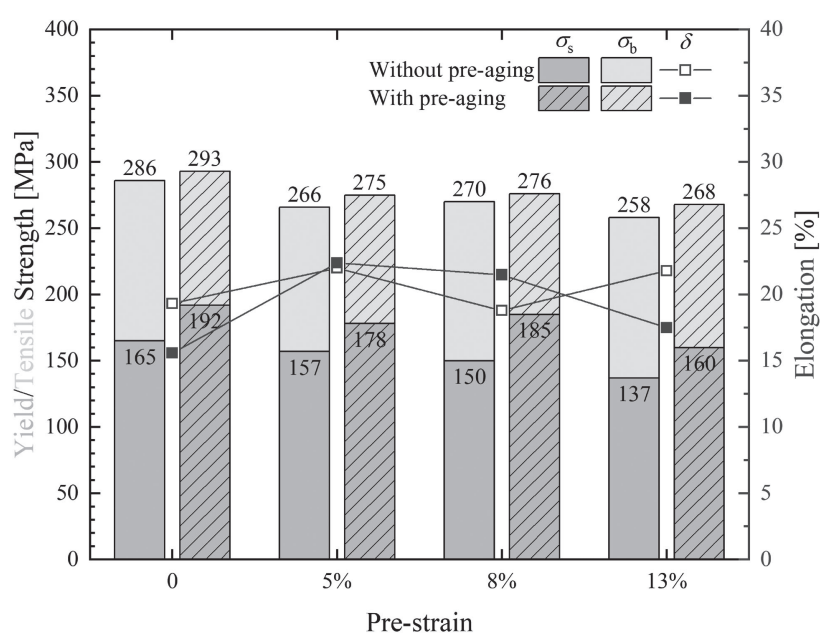

Fig. 4 Effect of pre-strain at elevated temperature and pre-aging on mechanical properties of AA6082 after paint-bake treatment.

combined suppressing effect of pre-strain at elevated temperature and pre-aging on the natural aging.

Figure 4 shows the effect of pre-strain at elevated temperature and pre-aging on mechanical properties of AA6082 after paint-bake treatment following the natural ageing. Compared with that in Fig. 3, $\sigma_{\mathrm{s}}$ and $\sigma_{\mathrm{b}}$ of samples subjected to pre-aging without pre-strain increase from 165 $\mathrm{MPa}$ and $286 \mathrm{MPa}$ to $192 \mathrm{MPa}$ and $293 \mathrm{MPa}$ after paint-bake treatment. It indicates that the pre-aging has a bake hardening effect. While with the pre-strain increases from $5 \%$ to $13 \%$, $\sigma_{\mathrm{s}}$ and $\sigma_{\mathrm{b}}$ of samples subjected to pre-strain without pre-aging after paint-bake treatment decrease little, meaning a weak bake softening effect. After introducing the pre-aging, $\sigma_{\mathrm{s}}$ and $\sigma_{\mathrm{b}}$ of samples increase. The pre-aging could compensate the strength decrease due to the bake softening. Figure 5 shows the TEM images of the samples with $5 \%$ pre-strain subjected to paint-bake treatment and pre-aging and sequent paint-bake treatment. A large number of dislocations are distributed in the alloy matrix after the $5 \%$ pre-strain, and most dislocations are entangled and pinned as shown in Fig. 5(a). The distribution and morphology of dislocations does not change 

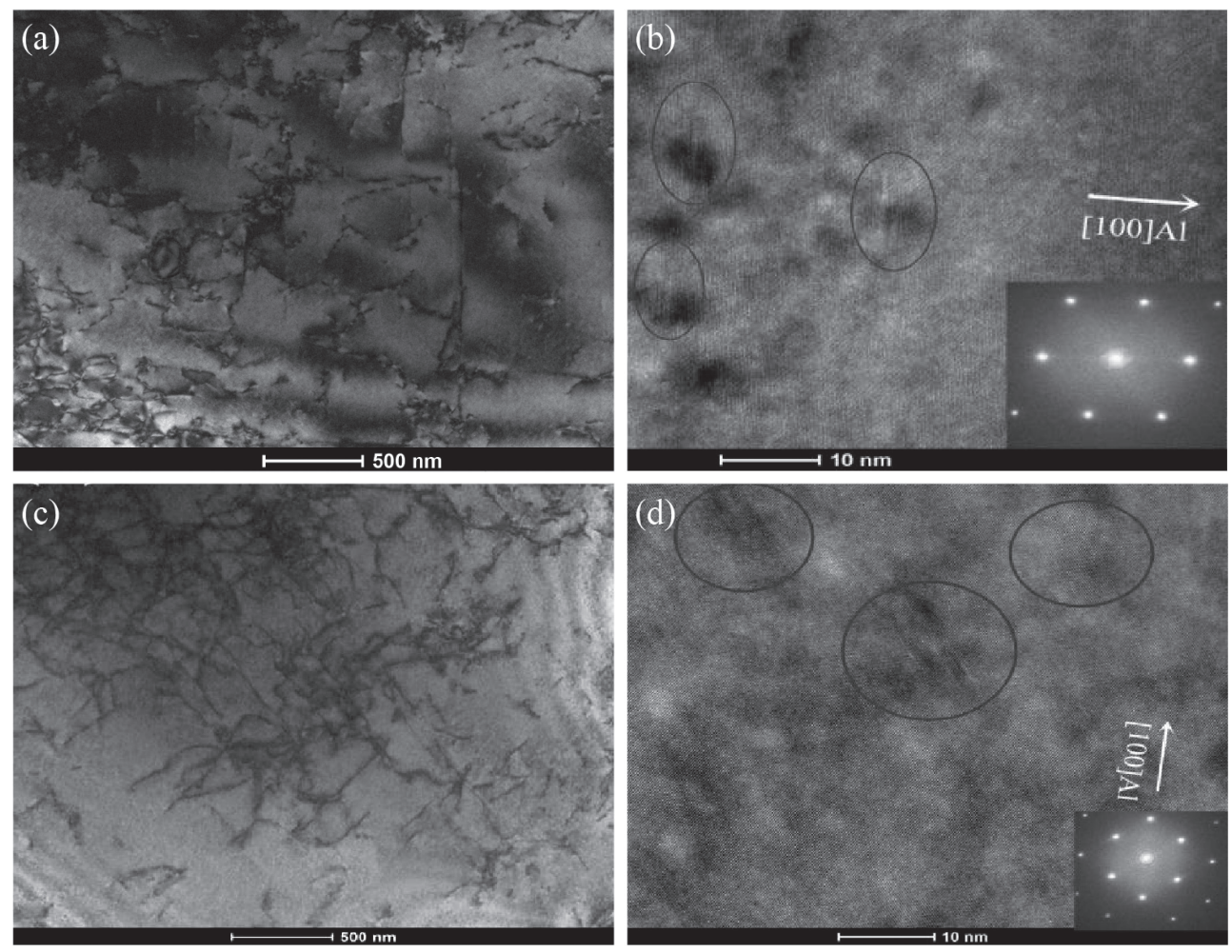

Fig. 5 TEM images of the samples with 5\% pre-strain subjected to (a), (b) paint-bake treatment and (c), (d) pre-aging and paint-bake treatment.

significantly after introducing the pre-aging as shown in Fig. 5(c), since the pre-aging temperature does not reach the recovery temperature of the alloy. By comparing Fig. 5(d) with Fig. 5(c), it can be seen that the precipitation phase increases and its size gets larger due to the pre-aging, resulting in the higher strength of the alloy. It is indicated that the GP zones or clusters formed during pre-aging are different from those formed during the natural aging, which are more stable and can act as nuclei for the precipitation of the main strengthening phase $\beta^{\prime \prime}$, and hence enhance the artificial aging response. ${ }^{13,14)}$

Figure 6 shows the effect of pre-strain at elevated temperature and pre-aging on mechanical properties of AA6082 after artificial aging following the natural ageing. $\sigma_{\mathrm{s}}$ and $\sigma_{\mathrm{b}}$ of samples subjected to pre-aging without pre-strain increase from $307 \mathrm{MPa}$ and $334 \mathrm{MPa}$ to $309 \mathrm{MPa}$ and $349 \mathrm{MPa}$ after the artificial aging. It indicates that the preaging has an active effect on artificial aging. While with the pre-strain increases from $5 \%$ to $13 \%, \sigma_{\mathrm{s}}$ and $\sigma_{\mathrm{b}}$ of samples subjected to pre-strain without pre-aging after artificial aging are below those without pre-strain, meaning pre-strain having detrimental effect on artificial aging. Dislocations induced by pre-strain at elevated temperature reduce the concentration of quenched-in vacancies and avoid clustering during the natural aging, while suppress homogeneous precipitation in the matrix due to the preferential formation of large precipitates on dislocation lines during the paint-bake treatment and artificial aging. It results in the aging softening. After introducing the pre-aging, $\sigma_{\mathrm{s}}$ and $\sigma_{\mathrm{b}}$ of samples increase. The pre-aging could compensate the softening due to the pre-strain.

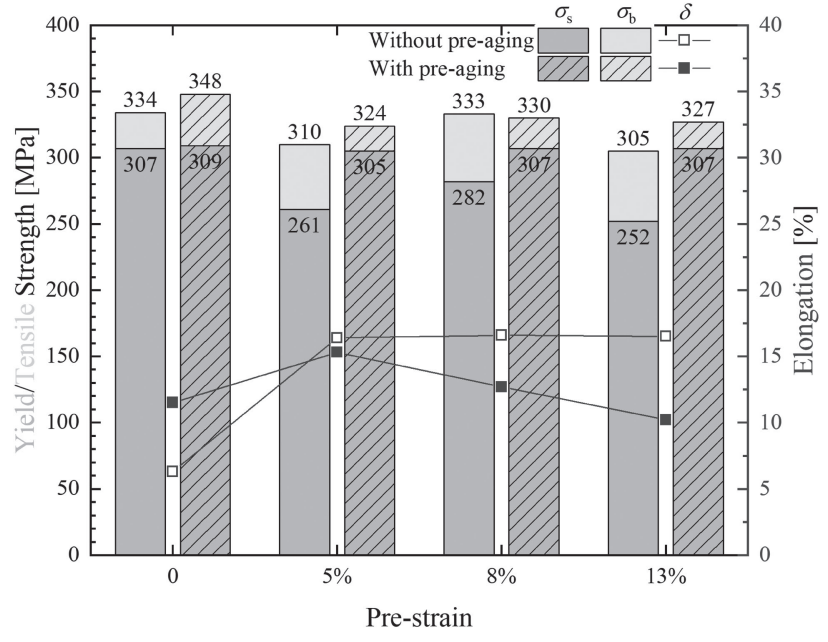

Fig. 6 Effect of pre-strain at elevated temperature and pre-aging on mechanical properties of AA6082 after artificial aging.

\section{Conclusion}

Sample sheets of AA6082-T6 were pre-strained at solution temperature in uniaxial tension at levels of $5 \%, 8 \%$ and $13 \%$, and then subjected to pre-aging at $140^{\circ} \mathrm{C}$ for 10 minutes, natural aging for two weeks, and paint-bake treatment at $180^{\circ} \mathrm{C}$ for 30 minutes or final artificial aging at $180^{\circ} \mathrm{C}$ for 6 hours, respectively. The yield strength, tensile strength and elongation of post-aged sheets were gained via standard uniaxial tensile tests. The microstructure analysis was carried out by TEM. Dislocations induced by pre-strain at elevated 
temperature reduce the concentration of quenched-in vacancies and avoid clustering during the natural aging, while suppress homogeneous precipitation in the matrix due to the preferential formation of large precipitates on dislocation lines during the paint-bake treatment and artificial aging. The GP zones or clusters formed during pre-aging can act as nuclei for the precipitation of the main strengthening phase during the paint-bake treatment and aging, and hence enhance aging response. The combined effect of pre-strain at elevated temperature and pre-aging results in that both of the pre-strain at elevated temperature and pre-aging suppress the natural aging of AA6082. Pre-strain at elevated temperature induces weak bake softening, and has a detrimental effect on artificial aging strengthening, while the pre-aging could compensate the strength decrease.

\section{Acknowledgments}

The work was supported by the National Natural Science Foundation of China (Grant No. 51505102).

\section{REFERENCES}

1) A. Afseth: Lightweight Design Worldwide 10 (2017) 12-15.

2) T.M. Lee, J.E. Carsley and S. Hartfield-Wunsch: SAE Int. J. Mater. Manuf. 4 (2011) 835-843.

3) S. Toros, F. Ozturk and I. Kacar: J. Mater. Process. Technol. 207 (2008) $1-12$.

4) E.S. de Argandoña, L. Galdos, R. Ortubay, J. Mendiguren and X. Agirretxe: Key Eng. Mater. 651-653 (2015) 199-204.

5) M. Kumar, N. Sotirov and C. Chimani: Mater. Sci. Forum 794-796 (2014) 796-801.

6) R.P. Garrett, J. Lin and T.A. Dean: Adv. Mater. Res. 6-8 (2005) 673680.

7) Q.Y. Yang, X.Y. Liu and X.Z. Fan: Mater. Trans. 60 (2019) 464-470.

8) X.F. Gu and T. Furuhara: Mater. Trans. 56 (2015) 917-922.

9) G.K. Quainoo and S. Yannacopoulos: J. Mater. Sci. 39 (2004) 64956502 .

10) K. Teichmann, C.D. Marioara, S.J. Andersen and K. Marthinsen: Metall. Mater. Trans. A 43 (2012) 4006-4014.

11) Y. Birol: Scr. Mater. 52 (2005) 169-173.

12) M. Kolar, K.O. Pedersen, S. Gulbrandsen-Dahl and K. Marthinsen: Trans. Nonferrous Met. Soc. China 22 (2012) 1824-1830.

13) W.F. Miao and D.E. Laughlin: J. Mater. Sci. Lett. 19 (2000) 201-203.

14) A. Serizawa, S. Hirosawa and T. Sato: Metall. Mater. Trans. A 39 (2008) 243-251. 